\title{
Kentron
}

Revue pluridisciplinaire du monde antique

19 | 2003

Le statut et l'image du corps dans la mythologie et la littérature grecques (suite et fin)

\section{Carmella Pirozzi, Il commo nella tragedia greca}

\section{François Jouan}

\section{(2) OpenEdition}

\section{Journals}

Édition électronique

URL : https://journals.openedition.org/kentron/1873

DOI : 10.4000/kentron.1873

ISSN : 2264-1459

Éditeur

Presses universitaires de Caen

\section{Édition imprimée}

Date de publication : 31 décembre 2003

Pagination : 255-257

ISBN : 2-84133-222-5

ISSN : 0765-0590

\section{Référence électronique}

François Jouan, «Carmella Pirozzi, /l commo nella tragedia greca », Kentron [En ligne], 19 | 2003, mis en ligne le 12 avril 2018, consulté le 13 octobre 2022. URL : http://journals.openedition.org/kentron/1873 ; DOI : https://doi.org/10.4000/kentron.1873

\section{(c) (i) $\odot$}

Creative Commons - Attribution - Pas d'Utilisation Commerciale - Pas de Modification 4.0 International - CC BY-NC-ND 4.0

https://creativecommons.org/licenses/by-nc-nd/4.0/ 
de chances d'être le plus fidèle au texte du grand poète: œuvre très construite, qui trouve son unité dans une structure qui se diffuse autour de l'épisode central du sacrifice de Ménécée, et dont l'unité n'est qu'apparemment bousculée par « une esthétique de la profusion »; le fait que ce drame soit un « clin d'œil à la tradition en même temps qu'un complet renouvellement de celle-ci » par une "stratégie du décalage » dans laquelle Euripide est passé maître, comme il est passé maître dans "l'intertextualité »; le fait également qu'Euripide ait voulu dans cette œuvre inaugurer « un nouveau type de théâtre, comme il le fera encore avec Oreste» (F. Jouan), théâtrepanorama dans lequel le spectacle prend une place primordiale, ces points ont conduit bon nombre de commentateurs, trop épris de rationalité, à méconnaître les qualités de cette œuvre. Sans parler de la notion de bon goût, qui a mené à des commentaires aberrants, et toujours bien sûr stérilisants, contre lesquels s'élève avec raison l'auteur (cf. son commentaire aux v. 1183-1185). Tout ceci pour dire que ce travail remarquable nous rend en plénitude ce drame d'Euripide, y compris dans son exodos si contestée, mais en nous donnant toujours avec honnêteté tous les éléments des problèmes et des discussions, qu'il s'agisse des scholies ou des commentaires modernes.

Grâce à cet ouvrage, bien rédigé, bien présenté et bien argumenté, le lecteur français dispose d'une somme le mettant à même d'apprécier en toute connaissance de cause cette tragédie exubérante du vieil Euripide.

Bernard Deforge

\section{Carmella Pirozzi, Il commo nella tragedia greca, Naples, M. d'Auria, 2003, 196 p.}

La définition du commos tragique que donne la Poétique d'Aristote, « un lamento [ $\theta \rho \hat{\text { vo }}$ ऽ] commun au chœur et à l'acteur », est lapidaire et en apparence sans équivoque. Pourtant, la grande variété des morceaux tragiques que l'on peut ranger sous cette étiquette ne répond pas à la simplicité de la définition, au point qu'il manquait à ce jour une étude d'ensemble qui distinguât clairement les seules parties de la tragédie qui méritent le titre de commos. Le terme même n'apparaît qu'une fois au v siècle, dans un vers des Choéphores (423): "J'ai frappé [sur ma poitrine] le commos arien.»

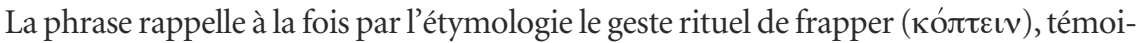
gnage physique du deuil, et son origine orientale. Mais même si les thrènes sont plus fréquents chez Eschyle que chez les deux autres tragiques, on ne peut suivre Wilamowitz qui voyait dans le commos le noyau originel de la tragédie, soit sous sa forme narrative (la déploration funèbre d'Andromaque au dernier chant de l'Iliade), soit sous sa forme poétique du goos, la lamentation lyrique. L'auteur soutient à juste titre 
qu'il s'agit d'une forme particulière, qui ne se confond ni avec le simple lamento, ni avec le chant amœbée, dans lequel se répondent deux acteurs. Malgré leur variété de fond et de forme, tous ces commoi offrent des traits communs : participation du chœur, sphère sémantique de la douleur et du deuil, avec référence aux gestes rituels de la déploration, vocabulaire spécifique, thèmes récurrents, technique précise dans l'alternance des voix et recours constant aux figures de rhétorique. $\mathrm{M}^{\mathrm{me}}$ Pirozzi distingue les commoi de forme pure (entièrement lyriques) et impure (combinés soit

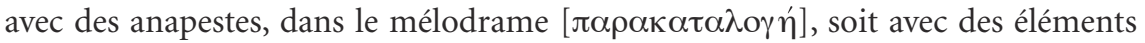
parlés, trimètres iambiques ou tétramètres trochaïques). Ils sont dits descendants si les éléments lyriques précèdent les parties parlées (épirrhéseis, plus souvent appelés épirrhèmes), ascendants dans le cas contraire. Leur place dans le drame n'est pas fixe, mais comme ils sont toujours liés à un crescendo émotif, ils se situent le plus souvent vers la fin de la pièce. Presque tous sont liés à une mort, qu'elle soit effective, proche ou ancienne, même mythique, ou encore imminente, voire seulement appréhendée, qu'elle concerne enfin une seule personne ou toute une communauté.

À partir de ces points communs, $\mathrm{M}^{\mathrm{me}}$ Pirozzi étudie successivement les 31 ensembles dans lesquels elle invite à reconnaître des commoi: on en compte 6 chez Eschyle (Perses, Agamemnon, Choéphores, 2 pour chacune de ces pièces) ; 6 aussi chez Sophocle (ils ne manquent que dans le Philoctète), 19 chez Euripide (Héraclides, Ion, Bacchantes, Iphigénie à Aulis en étant dépourvus, les autres pièces en comptant 1 ou 2, et les Suppliantes, 4). Ces examens en chaîne constituent le corps de l'ouvrage (p. 49163). Pour chaque morceau, l'auteur procède à une analyse formelle: structure (strophique ou astrophique, avec éventuellement des épodes), place des épirrhèmes pour les formes impures, de loin les plus nombreuses. Une analyse qui est loin d'être mécanique, mais implique une série de choix raisonnés, tant les métriciens sont divisés, parfois sur les limites, et souvent sur l'organisation interne des ensembles les plus complexes, mêlant la parole et le chant (par exemple, Antigone, 1261-1346, Hécube, 674-722, Andromaque, 1173-1225). Le grand mérite de cette étude est de ne jamais dissocier le fond et la forme, en reliant l'organisation formelle du morceau à l'expression des sentiments des choreutes et des personnages. Il arrive ainsi qu'il existe un décalage émotionnel profond entre le héros ou l'héroïne et le chœur (Cassandre et les vieillards de l'Agamemnon; Thésée et le chœur féminin de l'Hippolyte). Il y a des

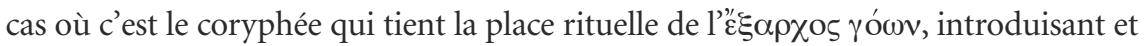
rythmant la lamentation de l'acteur : ainsi pour le chœur des vieillards avec Amphitryon, dans Héraclès. C'est au contraire Adraste qui joue ce rôle auprès des Mères dans les Suppliantes d'Euripide. Parfois encore le chœur a une mission surtout informative; plus rarement c'est le cas de l'acteur. De manière exceptionnelle, dans le commos de l'Oreste (1246-1310), la note funèbre est presque absente de la partie chorale, les jeunes femmes étant seulement hantées par la crainte de voir surgir des ennemis d'Électre et ses deux compagnons. L'intervention d'un personnage extérieur (comme 
Talthybios au cours du commos des Troyennes, 1246-1310) peut encore diviser le morceau en deux parties et en modifier le développement. Dans plusieurs drames de chacun des trois tragiques, deux des acteurs alternent leurs paroles ou leurs chants avec ceux du chœur. En d'autres termes, il y a presque autant de cas particuliers que d'exemples, ce qui montre la souplesse d'emploi de ce procédé et l'habileté des poètes à en adapter la forme au mouvement et au contenu dramatique de la pièce. L'étude des thèmes est ici toujours complétée par un examen approfondi du style et de la métrique, qui fournissent des moyens de mesurer l'intensité émotionnelle et ses variations au cours du commos: amplitude des procédés rhétoriques, fréquence des antilabai, nature du rythme (par exemple les dochmiaques de l'émotion intense ou les dactyles marquant l'apaisement, ou encore l'iambe, lié aux origines de la lamentation comme genre littéraire, la démarche semi-lyrique des séries anapestiques). Une analyse très technique en apparence, mais qui permet de pénétrer plus avant dans la genèse de la création poétique.

Trois brèves conclusions partielles tentent de caractériser la manière de chacun des grands tragiques dans leur emploi du commos. Elles préparent un bilan final, tracé en une vingtaine de pages. C'est surtout la communauté des thèmes qui fait du commos une structure de théâtre originale. Placé au moment où la tension émotionnelle est la plus forte, il est tout rempli de l'image de la mort, avec son contexte rituel de cris, de gémissements et de larmes. Thèmes les plus fréquents : la comparaison du passé et du présent, l'avenir tourné vers le désespoir, et la tentation de la mort. Le style et le rythme se modèlent sur la violence des sentiments. C'est dans ces morceaux que le pathos tragique peut atteindre son point extrême. Pour la forme, le mouvement au cours du temps va, comme on peut s'y attendre, vers l'assouplissement du schéma rigide initial, l'intégration plus marquée dans le développement dramatique et l'évolution psychologique des personnages, la localisation à des places variées de la pièce, le relâchement de la symétrie strophique au profit de formes libres, du type de la monodie, la proportion croissante des trimètres du parlé, encadrant ou divisant les parties chantées. Souvent situé dans l'exodos, le commos contribue, avec le "plaisir des larmes », à la catharsis tragique, conduisant le spectateur du pathos au pathèma.

Deux indices (textes anciens et auteurs modernes) terminent l'ouvrage. La recherche, qui était loin d'être facile, a été menée avec beaucoup de probité, d'indépendance d'esprit et toute la rigueur philologique qu'on pouvait attendre d'une élève du Pr. Garzya. On doit à $\mathrm{M}^{\mathrm{me}}$ Pirozzi une précise et utile définition d'une structure dramatique très particulière, liée à certaines des origines rituelles de la tragédie, mais qui porte en même temps témoignage de l'évolution des formes du lyrisme choral tout au long du v' siècle. 